\title{
Application of Quantitative 3D Microscopy to Understand Surface Properties of Engineering Thermoplastics
}

\author{
O. Guise, ${ }^{*}$ N. Preschilla, ${ }^{* *}$ D. Ellington, ${ }^{* * *}$ and A. Sikder ${ }^{* *}$ \\ * GE Industrial - Plastics, One Noryl Avenue, Selkirk, NY 12158, USA \\ ** GE India Technology Center, Hoodi Village, Whitefield Road, Bangalore 560066, India \\ ${ }^{* * *}$ GE Industrial - Plastics, One Lexan Lane, Mt Vernon, IN 47620-9367, USA
}

An integrated approach to SEM analysis, consisting of a new sample preparation method and 3D microscopy, is demonstrated as a proficient approach to evaluate and quantify surface properties of Engineering Thermoplastics (ETPs). ETPs find extensive applications in the automotive, consumer goods and medical device industries because of a property / processing balance allowing them to address a broad range of challenging requirements. ETPs having low surface gloss are materials of specific interest for automotive interiors, power tools, life fitness equipment etc. Subsequently, there is an increased need for information-rich rapid surface characterization methods to provide a mechanistic understanding of gloss reduction that would enable faster product and process optimization.

The ETP used in this study was a two-phase PC-Polyester blend containing impact modifiers and various additives such as antioxidants, fillers, hydro-stabilizers and photo-stabilizers. Three samples with different PC-Polyester ratios resulting in widely different gloss performance were selected for analysis. It was observed that the surface roughness measured with a profilometer showed a correlation to gloss values; higher surface roughness generally resulted in lower gloss values. Surface roughness is usually observed to have a strong correlation to surface morphology, especially the size and shape of the dispersed phase. Since gloss is a surface phenomenon, understanding this correlation through conventional methods involves multiple, tedious and timeconsuming microscopy, profilometry and composition analysis. This work reports how this complex task was successfully addressed through an integrated SEM method involving sample preparation by selectively etching out the dispersed PC phase with exposure to oxygen plasma followed by three-dimensional reconstruction of the surface and quantification with the MeX 3DSEM software package from Alicona. This etching method greatly simplified specimen preparation over techniques such as TEM and SPM and provided more accurate information on in-plane morphology. The 3D reconstruction method involved computation of the threedimensional surface structure from a set of three images for each specimen captured at different viewpoints obtained by eucentric tilting. The so-obtained three-dimensional dataset called a digital elevation model (DEM) forms the basis for further quantitative analysis.

Fig. 1(a) - (c) shows the SEM micrograph of a plasma-etched surface for the three samples chosen with varying PC-Polyester ratios. The morphology changed from well-dispersed domains to a large-scale co-continuous morphology; profilometry measurements performed independently show a steady increase in surface roughness with this change in morphology (not shown here). Conversely, 3D-SEM analysis of plasma exposed samples readily provided significantly superior visualization of the changes in surface morphology and roughness, as shown in the reconstructed 3D images from micrographs acquired at different tilt angles from the same location (Fig. 2 (a) (c)). Line profile analysis on these 3D reconstructions showed an increase in surface roughness 
consistent with profilometry data, as surface morphology changes from a finely dispersed phase to a large-scale co-continuous morphology. Surface roughness could also be quantified as the ratio of the true surface area of a specimen to its projected surface area; the ratio extracted from the DEM for each sample in Fig 2(a) - (c) was respectively 1.19, 1.46 and 1.57 (see Fig 3 (a)). Furthermore, the mean surface area - mean horizontal profile obtained after applying a roughness filter (acting like a high-pass filter) provided very high level information for deriving morphology - roughness - gloss correlations (Fig 3 (b)). In conclusion, this approach provided detailed insight into the dependence of roughness, mean surface area etc. on surface morphology and in turn gloss. Hence, it can also serve as a quick real-time quality tool for developing low gloss ETPs.
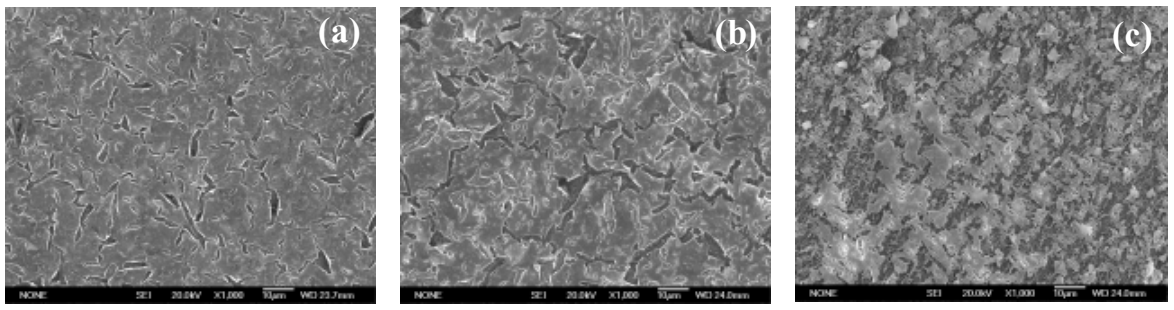

FIG. 1. SEM micrographs of plasma etched surfaces showing PC-Polyester dispersion with (a) finely dispersed discrete phase, (b) pseudo co-continuous morphology, and (c) co-continuous morphology.
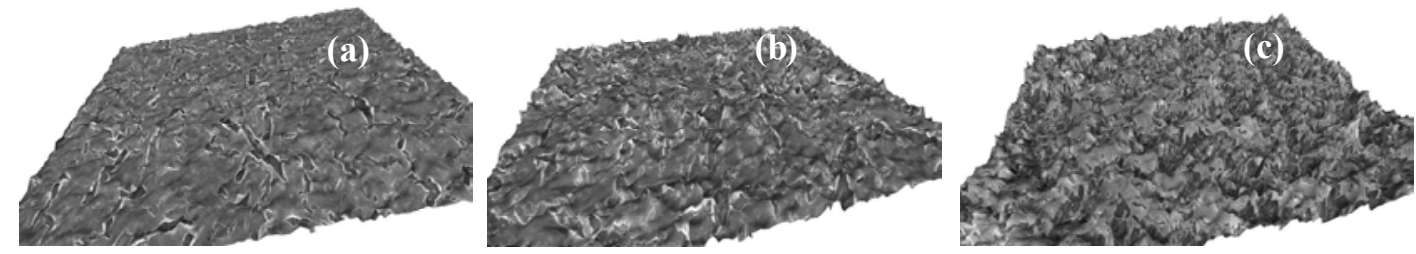

FIG. 2. 3D-SEM reconstruction of the respective samples shown in Fig 1(a) - (c). Steady increase in surface roughness with morphology change can be clearly visualized in these reconstructions. It should be noted that the z-scale was doubled in these images to help the reader visualize the increase of roughness.

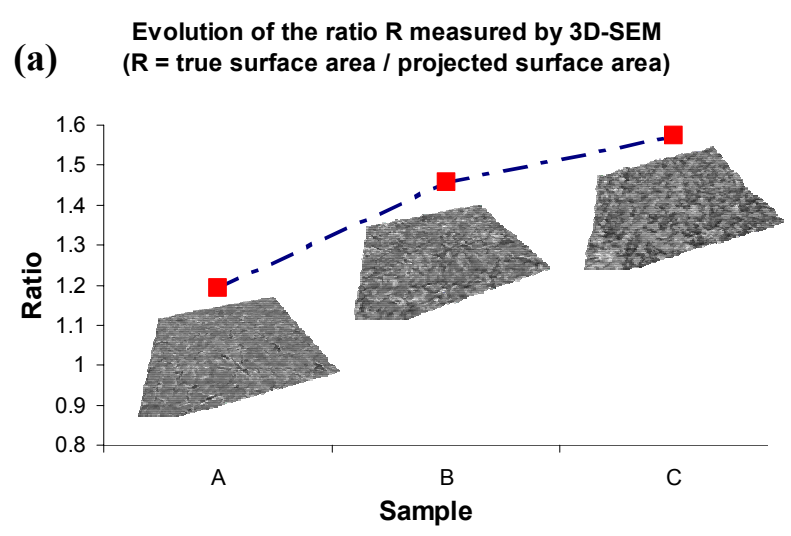

(b)

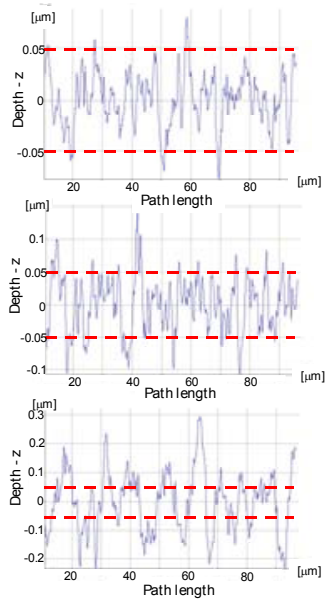

FIG. 3. (a) Surface area analysis - evolution of the ratio R (true surface area / projected surface area) measured by 3D-SEM. (b) Mean horizontal roughness profile for the three samples. The profile displayed is the mean of 100 profiles acquired through each image. The upper and lower red dashed lines delineate the $+0.05 \mu \mathrm{m}$ and $-0.05 \mu \mathrm{m}$ levels, respectively. 